\title{
Development and validation of a RAD-Seq target-capture based genotyping assay for routine application in advanced black tiger shrimp (Penaeus monodon) breeding programs
}

Jarrod L. Guppy ${ }^{1,2^{*}}$ (D), David B. Jones ${ }^{1,2}$, Shannon R. Kjeldsen ${ }^{1,2}$, Agnes Le Port ${ }^{1,2}$, Mehar S. Khatkar ${ }^{1,3}$, Nicholas M. Wade ${ }^{1,4}$, Melony J. Sellars ${ }^{1,4,5}$, Eike J. Steinig ${ }^{6}$, Herman W. Raadsma ${ }^{1,3}$, Dean R. Jerry ${ }^{1,2,7}$ and Kyall R. Zenger ${ }^{1,2}$

\begin{abstract}
Background: The development of genome-wide genotyping resources has provided terrestrial livestock and crop industries with the unique ability to accurately assess genomic relationships between individuals, uncover the genetic architecture of commercial traits, as well as identify superior individuals for selection based on their specific genetic profile. Utilising recent advancements in de-novo genome-wide genotyping technologies, it is now possible to provide aquaculture industries with these same important genotyping resources, even in the absence of existing genome assemblies. Here, we present the development of a genome-wide SNP assay for the Black Tiger shrimp (Penaeus monodon) through utilisation of a reduced-representation whole-genome genotyping approach (DArTseq).

Results: Based on a single reduced-representation library, 31,262 polymorphic SNPs were identified across 650 individuals obtained from Australian wild stocks and commercial aquaculture populations. After filtering to remove SNPs with low read depth, low MAF, low call rate, deviation from HWE, and non-Mendelian inheritance, 7542 highquality SNPs were retained. From these, 4236 high-quality genome-wide loci were selected for baits-probe development and 4194 SNPs were included within a finalized target-capture genotype-by-sequence assay (DArTcap). This assay was designed for routine and cost effective commercial application in large scale breeding programs, and demonstrates higher confidence in genotype calls through increased call rate (from $80.2 \pm 14.7$ to $93.0 \% \pm 3.5 \%$ ), increased read depth (from $20.4 \pm 15.6$ to $80.0 \pm 88.7$ ), as well as a 3 -fold reduction in cost over traditional genotype-by-sequencing approaches.

(Continued on next page)
\end{abstract}

\footnotetext{
* Correspondence: jarrod.guppy@my.jcu.edu.au; jarrod.guppy@jcu.edu.au 1 Australian Research Council Industrial Transformation Research Hub for Advanced Prawn Breeding, James Cook University, Townsville, QLD 4811, Australia

${ }^{2}$ Centre for Sustainable Tropical Fisheries and Aquaculture, College of Science and Engineering, James Cook University, Townsville, QLD 4811, Australia

Full list of author information is available at the end of the article
}

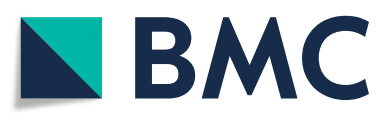

(c) The Author(s). 2020 Open Access This article is licensed under a Creative Commons Attribution 4.0 International License, which permits use, sharing, adaptation, distribution and reproduction in any medium or format, as long as you give appropriate credit to the original author(s) and the source, provide a link to the Creative Commons licence, and indicate if changes were made. The images or other third party material in this article are included in the article's Creative Commons licence, unless indicated otherwise in a credit line to the material. If material is not included in the article's Creative Commons licence and your intended use is not permitted by statutory regulation or exceeds the permitted use, you will need to obtain permission directly from the copyright holder. To view a copy of this licence, visit http://creativecommons.org/licenses/by/4.0/. The Creative Commons Public Domain Dedication waiver (http://creativecommons.org/publicdomain/zero/1.0/) applies to the data made available in this article, unless otherwise stated in a credit line to the data. 
(Continued from previous page)

Conclusion: Importantly, this assay equips the P. monodon industry with the ability to simultaneously assign parentage of communally reared animals, undertake genomic relationship analysis, manage mate pairings between cryptic family lines, as well as undertake advance studies of genome and trait architecture. Critically this assay can be cost effectively applied as $P$. monodon breeding programs transition to undertaking genomic selection.

Keywords: Genotype by sequencing, Advanced breeding, Black Tiger shrimp, Penaeus monodon, Diversity arrays technology, Aquaculture

\section{Background}

Genotype-by-sequencing (GBS) has quickly been recognised as a highly versatile and cost-effective approach to rapidly generate genome-wide marker data for an emerging aquaculture species, or those otherwise lacking existing SNP-based genomic resources [1]. In particular, since publication of the first Restriction-site Associated DNA Sequencing (RAD-Seq) protocol by Miller [2], a number of innovative developments of RAD-Seq protocols have been published. These include RAD [3], DArTSeq [4], ddRAD [5], 2bRAD [6], ezRAD [7] and 3RAD [8], all of which have seen dramatic improvements in the ease of generating reliable, repeatable and low-cost genotype data through GBS methods across a plethora of species (reviewed by [9]). As such RAD-Seq protocols have been increasingly applied within a number of aquaculture and fisheries fields of research (reviewed by $[1,10]$ ).

Across the range of RAD-Seq methods now available, each protocol contains its own subtle differences in methods undertaken throughout the sample-tosequencing process, which have been reviewed in detail by Puritz [11], Andrews [9] and Robledo [1]. Importantly however, all commonly utilized methods (e.g. RAD, [3]; ddRAD, [5]; DArTSeq, [4]) share the fundamental approach of sequencing only a reduced representation of each individuals' genome. Specifically, by undertaking genomic complexity reduction steps (i.e. restriction enzyme digestion and/or fragment size selection) nextgeneration sequencing efforts can be focused more efficiently by consistently sequencing specific regions across each individuals' genome [9].

In an effort to further ensure consistent sequencing of the same genomic regions, a number of RAD-Seq methods have been paired with 'targeted sequence capture' protocols to enrich desired sequences (known as 'RAD-tags') in the sequencing libraries before final preparation for high-throughput next-generation sequencing [12]. Specifically, these approaches [e.g. Rapture, [13]; hyRAD, [14]; DArTCap, unpublished modifications of Sansaloni [4]] utilize bead-based hybridization (e.g. DYNAbeads ${ }^{\circ}$ ) or capture baits (e.g. MYbaits ${ }^{\circ}$ ) to exclusively select RAD-tags from DNA samples that have already undergone previous traditional complexity reduction steps. This second stage of library refinement is referred to as 'enrichment' [12].

By following this two-stage library preparation approach, it is possible to further improve the consistency of genotype data compared to traditional RAD methods in two ways. Firstly, it is possible to obtain higher sequence read coverage of a refined set of loci which improves confidence in genotype calls [13]. Secondly, through multiplexing more samples within a fixed allocation of sequencing effort, it is possible to substantially reduce the genotyping cost per individual $[9,13,15,16]$. By leveraging these additional strengths of 'RAD-Seq target-capture' hybrid protocols, genotyping strategies (i.e. adjusting optimal sequence depth and/or multiplexing a higher number of individuals) can be tailored to efficiently fulfil the intended uses of genotype data in aquaculture (i.e. tracing pedigree, allocating matepairings and determining family contributions). Furthermore, when 'RAD-seq target capture' genotyping is coupled with the collection of large phenotypic datasets, a plethora of advanced applications can be achieved. The calculation of genomic relationship matrices (GRM), genomic estimated breeding values (GEBV); discovery of selection signatures, implementation of genomic selection (GS), genome-wide association studies (GWAS), quantitative trait loci mapping (QTL), and genetic marker imputation are now common place in the study and management of most terrestrial livestock species.

Black tiger shrimp (Penaeus monodon) are an aquaculture species of significant value ( $\sim$ SUSD 4.5 billion [17]; ), however, the industry has been troubled by inconsistent seedstock quality and numerous devastating disease outbreaks. While producing a current global production of 713,318 metric tonnes per annum [17], the industry stands to benefit greatly from developing genetic tools to manage existing breeding programs more effectively, and furthermore, facilitate a transition to genomic based breeding programs (i.e. genomic selection [18-21];). While efforts have been undertaken to develop a range of genomic markers (e.g. microsatellites [22-24]), AFLPs [25], low-density (59-122) SNPs [26]), these marker panels lack the power for applications required in advanced breeding programs particularly when considering complex traits like pathogen resistance or tolerance [20, 
26, 27]. The only existing medium density SNP-based marker panel (6000 SNPs) produced for black tiger shrimp was developed upon the high-cost Illumina iSelect array platform, and as we are aware, has yet to be made accessible for commercial use [28]. To date, no genotyping assay has been produced for black tiger shrimp that can feasibly be applied in routine highvolume applications or to service the industries desire to progress towards advanced selective breeding programs.

Here, the processes and rational underlying the development of a hybrid 'RAD-Seq target-capture' GBS assay for application within a black tiger shrimp industrial aquaculture setting are detailed. We demonstrate the versatility of this assay through traditional parentage assignment of animals reared under communal commercial conditions (i.e. pedigree unknown), as well as the utility of this assay in GRM calculations which set the foundations for accurate estimations of GEBVs. The ability to generate accurate GEBVs is integral for the establishment of genomic selection programs in black tiger shrimp.

\section{Results}

\section{SNP discovery, quality assessment and selection}

After processing raw sequencing data from a DArTseq GBS library of 650 individuals (ten individuals were excluded during library preparation), a dataset of 24,683 RAD-tags containing 31,262 SNP markers (31 K SNP dataset), was returned. An average of 1.37 SNPs $\pm 0.6 \mathrm{SD}$ were present in each RAD-tag, with a maximum of six SNPs observed in a single RAD-tag (19,288 tags with one SNP, 4300 tags with two SNPs, 915 tags with three SNPs, 155 tags with four SNP, 22 tags with five SNP, three tags with six SNPs). For this raw $31 \mathrm{~K} \mathrm{SNP} \mathrm{dataset,}$ the average genotype call rate was $0.86 \pm 0.14 \mathrm{SD}$ and the average MAF was $0.11 \pm 0.15 \mathrm{SD}$.

An average of $\sim 2.5$ million reads were allocated to each individual in library preparation and sequencing; however, after removal of low quality sequences, monomorphic loci, and SilicoDArT markers (presence/absence variants), an average of $459,987 \pm 88,493$ SD reads were associated with each individual (366,609,683 total reads over 650 individuals). The average read depth over all non-missing genotype calls was 17.0 $\pm 18.3 \mathrm{SD}$, however, $27.0 \%$ of non-missing genotype calls had 5 or less reads associated.

To ensure only the highest quality markers were available for baits probe selection and assay development, a series of SNP filtering thresholds were implemented to remove individual genotype calls with low confidence, remove less informative markers and remove erroneous data (Fig. 1). Filtering for minimum read depth $(<5)$ removed $20.4 \%$ of the non-missing genotype calls $(4,155$, 990), increasing the overall missing-ness of the $31 \mathrm{~K}$

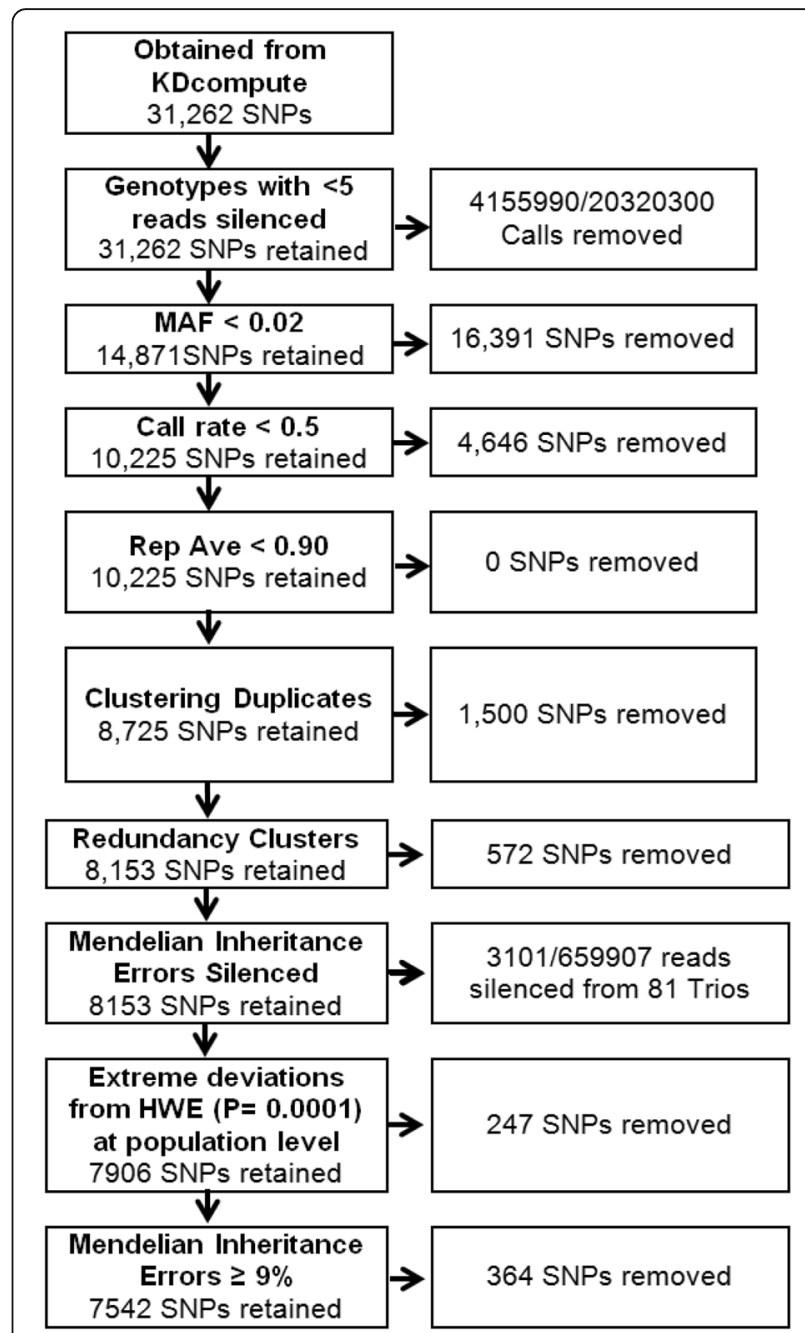

Fig. 1 SNP qualtity control pipeline for development of Peneaus monodon genotyping assay and the number of SNPs retained after each step of filtering

dataset from 14 to $33 \%$. Subsequently filtering for minimum minor allele frequency of $\geq 0.02$ removed 16,391 SNPs. A further 4646 SNPs were removed due to having a minimum call rate equal to or below 0.5 ; no SNPs were discarded due to having a repeatability score of less than 0.9 due to pre-filtering on repeatability before data was provided. For SNPs that were derived from identical clones (100\% rad-tag sequence similarity), the SNP with the highest MAF was retained from each clone resulting in the removal of 1500 SNPs. Similarly, redundancy clustering of clone sequences at $95 \%$ sequence similarity removed a further 572 SNPs derived from highly similar clones. Of the markers retained, tests of conformity to Mendelian inheritance patterns and HWE were conducted. A total of 364 SNPs were removed due to MI errors $(>9 \%)$ and 247 SNPs were removed due to HWE deviations. Finally, 3101 genotype calls identified as MI errors across the remaining SNPs were silenced (Fig. 1). 
In total 23,720 SNPs were removed from the $31 \mathrm{~K}$ dataset, leaving 7542 high quality SNPs for further ranking and final SNP selection. After filtering, the average MAF increased from $0.11 \pm 0.15$ to $0.22 \pm 0.15$. While the average per genotype call rate decreased from $0.86 \pm$ 0.14 to $0.84 \pm 0.14$, the average read depth per genotype call increased from $17.0 \pm 18.2 \mathrm{SD}$ to $30.7 \pm 27.9 \mathrm{SD}$. Furthermore, the rate of average Mendelian inheritance errors detected across SNPs decreased from 3.2 to $1.4 \%$ after filtering.

\section{SNP number and GRM analysis}

To determine the number of markers required for accurate genomic relationship calculations and selection of the density of the DArTcap panel, a number of marker subsets were modelled. An increasingly consistent GRM estimate was achieved by including progressively more random markers from 100 to 1000 markers (Fig. 2). With 1000 markers a correlation of 0.95 was consistently achieved when compared to the full 75,442 marker set (Fig. 2). Increasing the number of markers from 1000 to 4000 further increased the correlation between marker sets, with the correlation exceeding the desired minimum cut off for future assay applications of 0.98 at 4000 markers (Fig. 2).

Since GRM correlations indicated an optimal assay size of 4000 SNPs, a QC score was assigned to the set of 7542 high quality DArTseq markers allowing the prioritisation of 4236 SNPs for DArTcap probe systhesis. For this selected subset of markers, the average call rate was $80.2 \% \pm 14.7 \%$, MAF was $0.35 \pm 0.28$ and the average read depth per non-missing genotype call was $20.4 \pm$ 15.6. Furthermore, genomic relationship values calculated with the 4236 markers showed high correlation $\left(r^{2}=0.987\right)$ to the full 7542 marker panel (Fig. 3a). A number of pairwise relationships were estimated to be negative. These values are a result of the distinct underlying population structure between individuals from East Australina Coast populations and those from Northern Territory populations [29].

\section{Linkage disequilibrium}

Limited evidence of substantial linkage disequilibrium was observed with the DArTseq datasets. A total of 4 marker pairs were found to be linked $\left(r^{2}>0.80\right)$ in the 7542 SNP dataset when considering all available individual genotypes, while only two of these marker pairs persisted within the selected 4236 SNPs $\left(r^{2}\right.$ values of 0.96 and 0.85 ). When assessing genotypes of the Northern Territory individuals only (Tiwi Island, Joseph Bonaparte Gulf, Gulf of Carpentaria) from the 7542 SNP dataset, there were three marker pairs with $r^{2}$ values greater than 0.8. Likewise, for East Australian Coast individuals only (Bramston Beach, Townsville, Etty Bay), there were $13 r^{2}$ values greater than 0.80 , however, only one of these was also observed in the Northern Territory population. Using only Northern Territory individuals from the 4236 SNP dataset, there was one marker pair identified to be

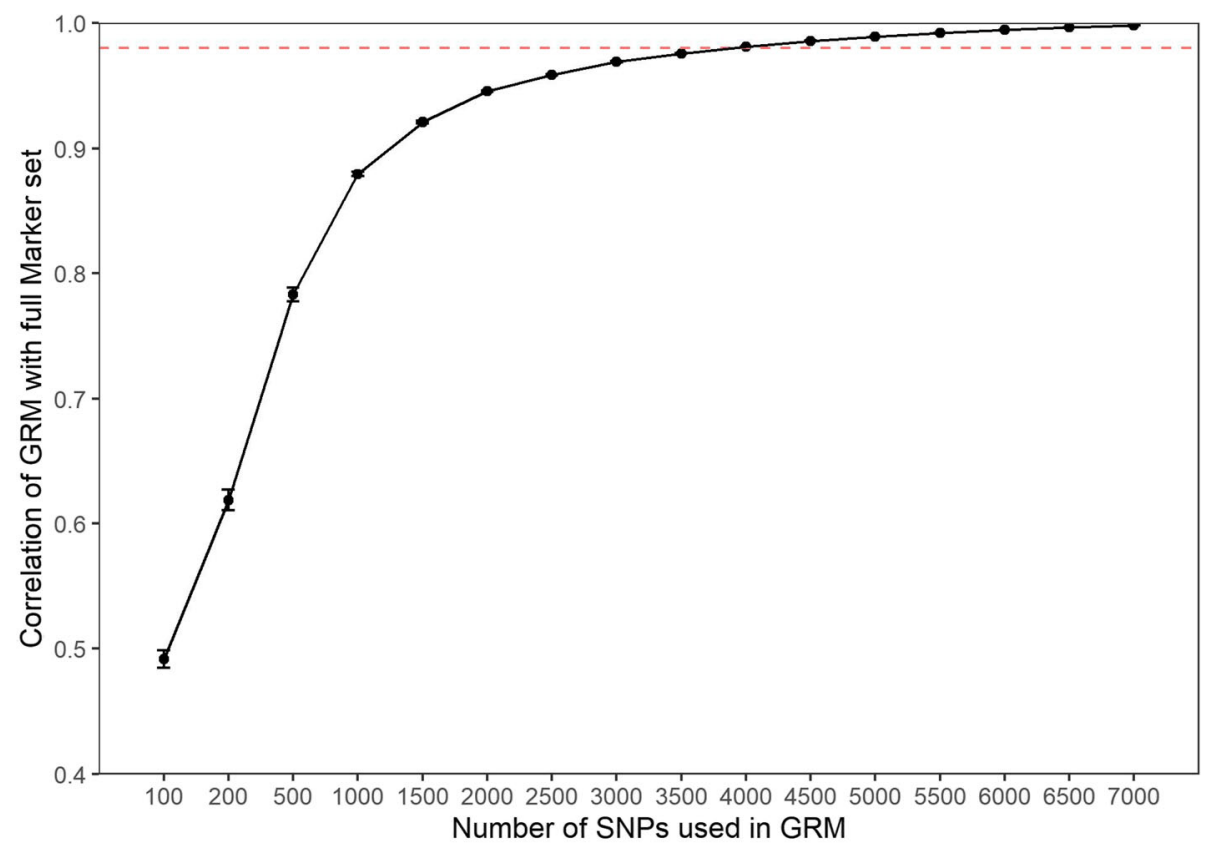

Fig. 2 Correlations between GRMs estimated from randomly selected subsets of $n$ marker density $\left(G_{n i}\right)$ and the complete pool of available markers (7542 SNPs). Average correlations and error (SE) of each $n$ derived from 50 replicated GRM estimates. Desired correlation of $>0.98$ indicated by the dashed line 


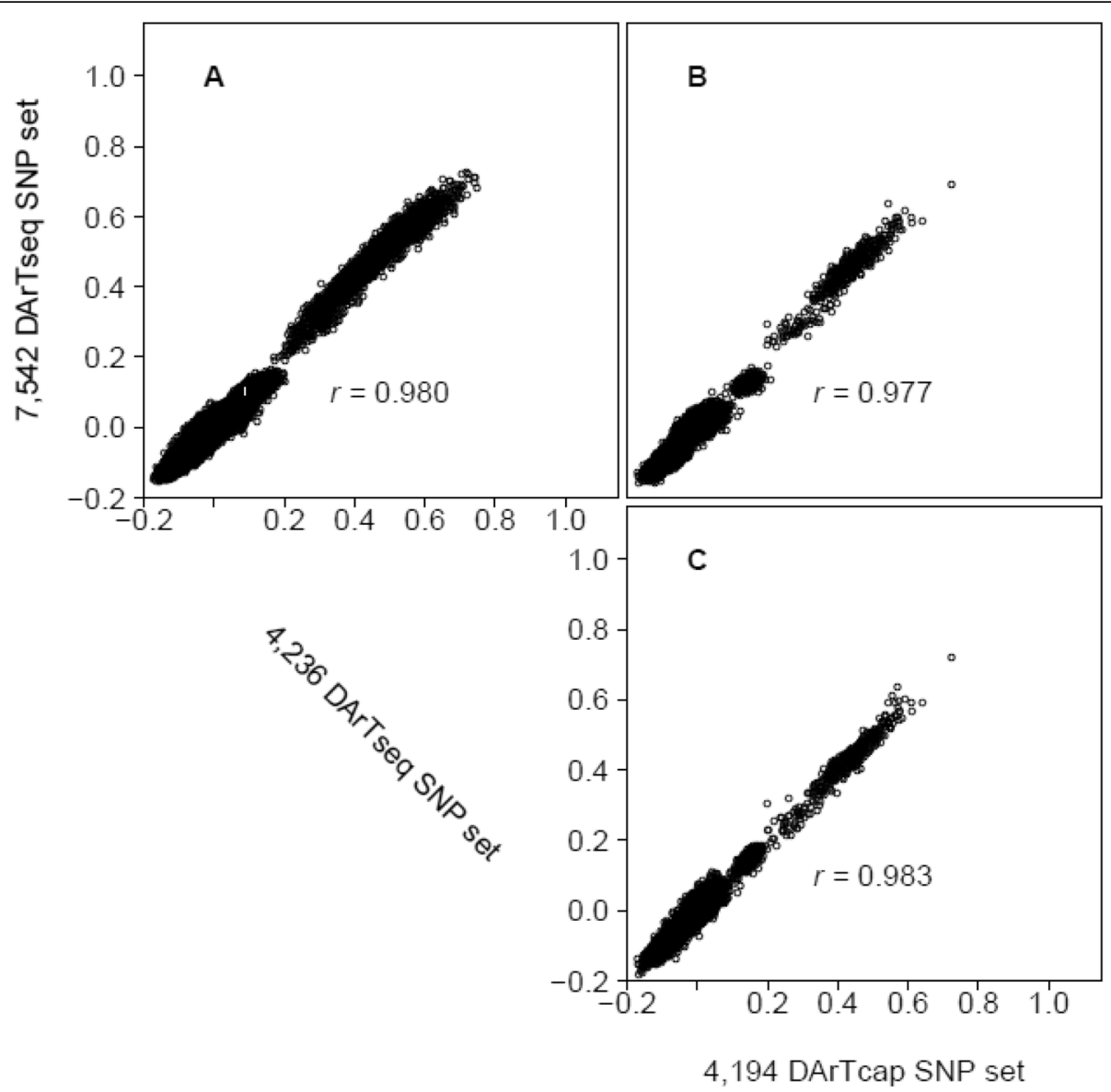

Fig. 3 Comparison of genomic relationship values calculated from the full 7542 SNP set, the selected 4236 SNP set provided for DArTCap probe synthesis, and the final set of 4194 DArTcap SNPs. GRMs were calculated with all common individuals available between datasets including; 650 individuals (a), 195 individuals (b) and 195 individuals (c) respectively

in $\mathrm{LD}\left(r^{2}>0.80\right)$. Likewise, when considering genotypes of East Australian Coast individuals, there were $12 r^{2}$ values greater than 0.80 , with one pair shared with those observed in the Northern Territory population.

\section{DArTcap assay validation}

A number of samples resubmitted for genotyping did not pass sample digestion $\mathrm{QC}$, and were excluded from sequencing (Table 1). This was most prominently

Table 1 Individuals genotyped with DArTseq and DArTcap

\begin{tabular}{|c|c|c|c|c|c|}
\hline \multirow[t]{2}{*}{ Population } & \multirow[t]{2}{*}{ Region } & \multicolumn{2}{|l|}{ DArTSeq } & \multicolumn{2}{|l|}{ DArTCap } \\
\hline & & \# submitted & \# passing library preparation & \# submitted & \# passing library preparation \\
\hline Townsville & East Coast, Australia & 22 & 22 & 10 & 10 \\
\hline Etty Bay & East Coast, Australia & 50 & 50 & 15 & 7 \\
\hline Bramston Beach & East Coast, Australia & 60 & 60 & 12 & 9 \\
\hline Gulf of Carpentaria & Northern Territory, Australia & 42 & 35 & 14 & 14 \\
\hline Tiwi Island & Northern Territory, Australia & 56 & 56 & 10 & 10 \\
\hline Joseph Bonaparte Gulf & Northern Territory, Australia & 34 & 34 & 13 & 13 \\
\hline Nickol Bay & Western Australia, Australia & - & - & 19 & 18 \\
\hline 1st Generation & Farm Stock & 165 & 162 & 87 & 86 \\
\hline 2nd Generation Set 1 & Farm Stock & 231 & 231 & 90 & 46 \\
\hline 2nd Generation Set 2 & Farm Stock & - & - & 282 & 272 \\
\hline Total & & 660 & 650 & 552 & 485 \\
\hline
\end{tabular}


observed in the initial set of 2nd generation farm stock (44 of 90 submitted), and was identified to be due to DNA degradation during storage. Across the remaining 462 individuals, 21 samples from across various populations failed QC and were excluded (Table 1). Processing of raw sequencing data obtained from a DArTcap target-capture library including a total of 485 individuals produced a dataset of 15,880 RAD-tags containing a total of 26,751 SNP markers (raw DARTcap dataset). An average of 1.7 SNPs \pm 1.0 SD were present in each RADtag, with a maximum of 18 SNPs observed in a single RAD-tag (9110 tags with 1 SNP, 4142 tags with 2 SNPs, 1731 tags with 3 SNPs, and 897 with $>3$ SNPs). For the raw DArTcap dataset, the average individual call rate was $72.0 \% \pm 6.8 \% \mathrm{SD}$, average genotype call rate was $72.0 \% \pm 25.17 \%$ SD, and average MAF was $0.11 \pm 0.13$ SD. Approximately 600,000-700,000 reads were allocated to each individual in library preparation and sequencing, and after removal of low quality sequences, an average of $670,610 \pm 185,993$ reads were associated with each individual $(328,598,913$ total). The average read depth per genotype call was $34.8 \pm 61.8 \mathrm{SD}$, however $32.0 \%$ of non-missing genotype calls had 5 or less reads associated.

Within the raw DArTcap dataset, 4194 (99.0\%) of the selected 4236 panel were observed, with only 52 baits probes failing to capture the desired rad-tag sequences. For the 4194 DArTcap dataset, the average individual call rate was $93.0 \% \pm 3.5 \% \mathrm{SD}$, and average MAF was $0.23 \pm 0.15$ SD. An average of $312,343 \pm 83,235$ reads were associated with each individual $(153,048,102$ total). An average of $46.8 \% \pm 1.6 \%$ of the total DArTcap sequencing effort was successful in obtaining target sequences per individual. The average read depth per nonmissing genotype call was $80.0 \pm 88.7 \mathrm{SD}$, while $5.78 \%$ of non-missing genotype calls had 5 or less reads associated.

\section{DArTcap assay utilisation Parentage assignment}

The results of parentage assignment success using Colony are presented in Table 2 . At a conservative genotyping error rate of $10 \%$, parentage assignment across the three sets of markers (7542 DArTseq, 4236 DArTseq,
4194 DArTcap) were essentially identical, where only one individual (from 72 known parent-progeny relationships) had a single parent incorrectly left unassigned (false exclusion) when using the 4194 DArTcap marker set (1.39\% assignment error). The occurrence of false exclusions increased to 4.2 and $8.3 \%$ for the DArTcap marker set when using genotyping error rates of 5 and $1 \%$ respectively. The rate of false exclusions (2.3\%) remained consistent irrespective of the error rate used in Colony for both the 7542 and 4236 DArTseq marker sets; however, the individual false exclusions did vary between either exclusion of the mother or father for some progeny.

Across all analyses, progeny containing higher missing data $(>10 \%)$ accounted for the majority of false parent exclusions. Furthermore, irrespective of the markers used in the analysis, there was a single individual where at least one of the two true parents could not be assigned consistently. For this individual, across analyses the unassigned parent was not consistent with either both parents unassigned, only the mother unassigned, or only the father unassigned. There were no untrue parent assignments (false assignments) observed across any of the assignment analyses, with all putative parents correctly excluded where the true parent/s were absent from the dataset tested.

\section{Genomic relationship calculations}

Genomic relationship values calculated with the 4194 DArTcap markers showed high correlation to the 7542 DArTseq marker set $\left(r^{2}=0.98\right.$; Fig. $\left.3 \mathrm{~b}\right)$ and the 4236 DArTseq marker panel $\left(\left(r^{2}=0.98\right.\right.$; Fig. 3c), importantly indicating high concordance between the genotypes obtained for the 195 samples that were genotyped through the two GBS approaches. As with the comparision of DArTseq 7542 and 4236 marker panels (see above) a number of pairwise relationships were less than 0 (no relationship). This is likely to be due to the structured nature of the wild samples included in the dataset $[29,31$, 32].

To further explore the utility of the 4194 DArTcap SNP panel, genomic relationship values were calculated for an additional independent set of G2 samples ( $n=$ 272) that were produced from novel Northern Territory

Table 2 Success rate of parentage assignment analysis using three SNP marker sets (7542 DArTseq, 4236 DArTseq, 4194 DArTcap) and three genotyping error rates that range from strict to conservative $(0.01,0.05$ and 0.1$)$ in Colony [30]. There were no untrue parent assignments for any dataset at any error rate

\begin{tabular}{llll}
\hline SNP dataset & \multicolumn{3}{l}{ Success rate of parentage assignment at different genotyping error rates } \\
\cline { 2 - 4 } & 0.01 & 0.05 & 0.10 \\
\hline 7542 DArTseq & $97.2 \%$ & $97.2 \%$ & $97.2 \%$ \\
4236 DArTseq & $97.2 \%$ & $97.2 \%$ & $97.2 \%$ \\
4194 DArTcap & $91.8 \%$ & $95.8 \%$ & $98.6 \%$ \\
\hline
\end{tabular}


sourced broodstock (i.e. not genotyped with either DArTseq or DArTcap). Utilising the GRM values of progeny alone, in the absence of parental genotypes, it is possible to clearly obtain sib-ship information (block structure in Fig. 4), including delineating full-sib and half-sib relationships. Furthermore, when compared to routine pedigree based relationship matrixes, it is possible, by assessing the values off the diagonal of the heatmap (within and outside blocks), to identify cryptic relatedness between otherwise traditionally unrelated individuals (Fig. 4).

\section{Population segregation}

The ability of the 4194 DArTcap SNP array to segregate samples obtained from different populations across the Australian distribution of $P$. monodon was assessed by comparing these individuals through discriminant analysis of principle components (DAPC). By comparing the first two principle components (with PC1 explaining $52.8 \%$ and PC2 explaining $44.3 \%$ of the variation), three distinct clusters of individuals are clearly identified (Fig. 5). Specifically, samples obtained from within East Australia Coast, Northern Territory and Western Australia form their own clusters. The G1 broodstock and G2 progeny also cluster with their source population (Northern Territory) showing no clear separation as a result of breeding practices (Supplementary Figure 2). The associated regional structure is consistent with previously identified population structure [22].

\section{Discussion}

Black tiger shrimp aquaculture is of substantial economic value globally and is forecast to expand rapidly in Australia with consistently increasing demand in domestic and international markets. However, the industry as a whole has lacked a viable low-cost genotyping assay capable of being used for advanced selective breeding programs, including genomic selection. In this study, we undertook de-novo SNP discovery, marker quality control and filtering, before selecting and successfully validating a custom DArTcap genotyping assay containing 4194 SNPs across Australian populations of P. monodon. Access to such assays is highly sought after within $P$. monodon breeding programs, particularly to facilitate the transition to genomic selection.

\section{DArTcap assay development SNP discovery}

In this study, we obtained 31,262 SNPs (24,683 unique RAD-tags) by employing de-novo SNP discovery (DArTseq) genotyping of 650 individuals collected from across the natural range of Australian P. monodon [22]. The number of markers (and RAD-tags) obtained in this study are closely comparable to studies using $2 \mathrm{~b}-\mathrm{RAD}$ [33] and SLAF-seq [20] in white leg shrimp (Litopenaeus vannamei) with 25,140 SNPs, and 23,049 SNPs obtained previously. A recent study using SLAF-seq for P. monodon obtained a lower total number of markers (6821) than this study, however, these were reported after

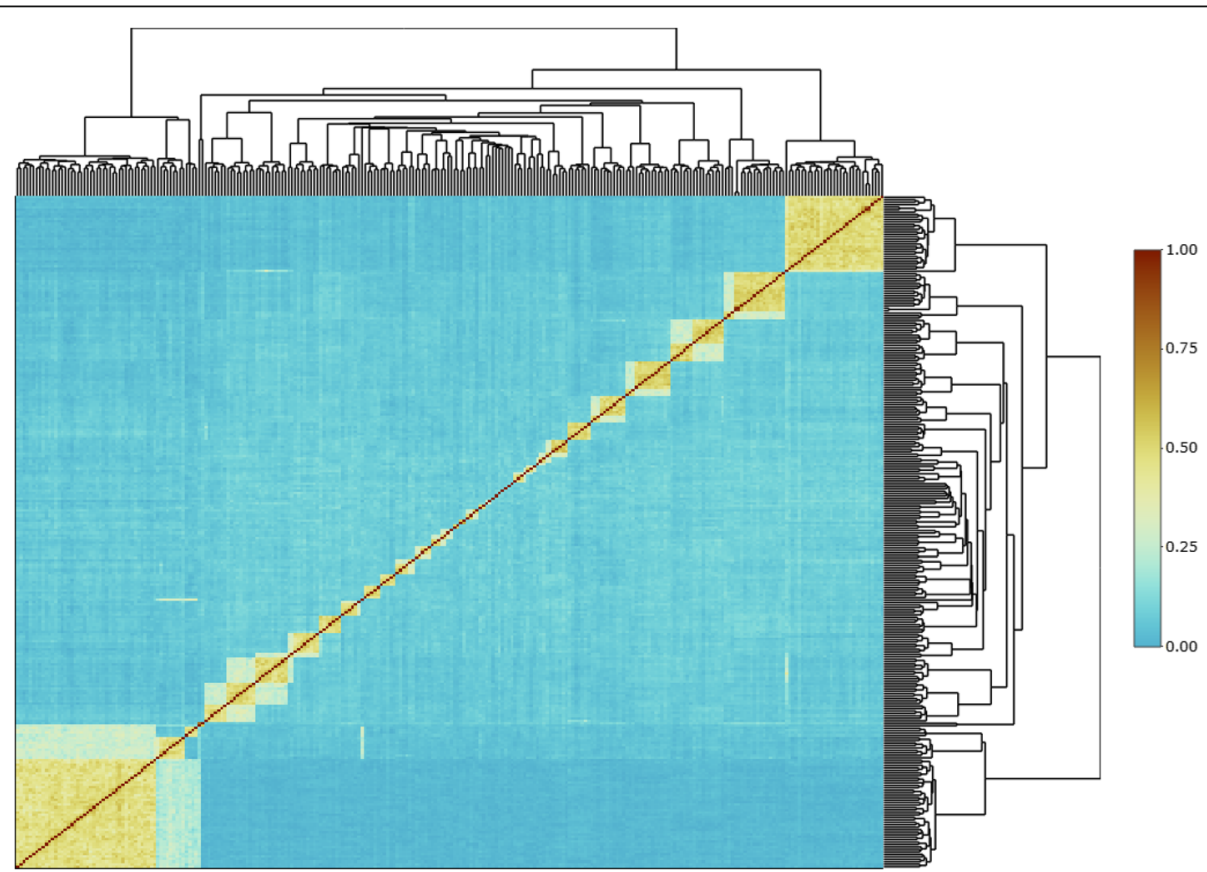

Fig. 4 Heat-map with dendrogram clustered from a genomic relationship matrix (GRM) of commercial, communally spawned black tiger shrimp progeny. The pixel colouring denotes proportion of genomic relationship between two individuals with $0=$ no relationship and $1=i d e n t i c a l$. Plotted with R package heatmaply 


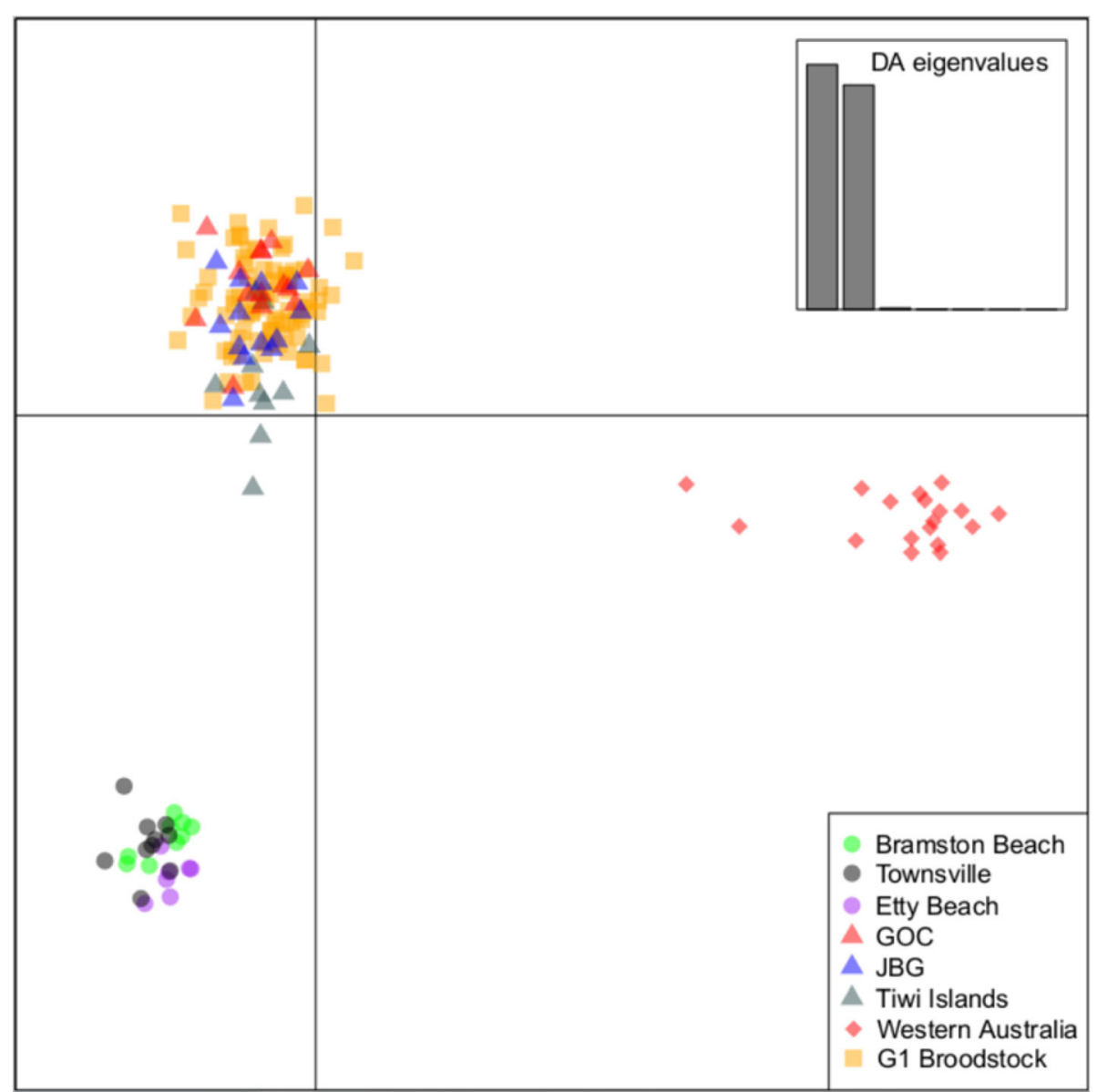

Fig. 5 Clustering of 168 wild-sourced samples based upon genetic similarity shown through discriminant analysis of principle components (DAPC). PC1 and PC2 are shown on the $x$ and $y$ axis respectively. PC1 explains $52.8 \%$ and PC2 explains $44.3 \%$ of the variation

completion of filtering (markers retained based on Parent read depth (10-200), 90\% call rate, MI >0.01) and as such is not directly comparable. Furthermore, these markers were utilised for linkage map construction, rather than with the intent of inclusion in a genotyping assay, and as such a single large family ( $98 \mathrm{G} 2$ progeny, G0 stock derived from Mozambique) was used in SNP discovery [34] rather than employing a diverse discovery population. Previously, Baranski [28] utilised transcriptome sequencing (RNA-seq) of $P$. monodon obtained from four locations around the coast of India, to discover 473,620 SNPs. Baranski [28] proceeded to produce a custom solid-state $6 \mathrm{~K}$ Illumina iSelect genotyping assay, and a subsequent genetic linkage map with the completed assay, which has been further utilised in GWAS and QTL studies [35]. In this study we aimed to produce a low-cost genotyping assay that relied upon genotype-by-sequencing (requiring a restriction enzyme complexity reduction), and therefore were not able to directly integrate existing SNP resources from Baranski [28]. Furthermore with the intended focus on utilisation of the DArTcap assay within the Australian industry, it was important to derive SNPs from Australian stock, to ensure marker 'informativeness' was maximised.

\section{Determining SNP density required for GRM analysis}

Since the initial shift from traditional co-dominant markers (e.g. microsatellites) to SNPs, there has been a focus on increasing the density of SNPs included in assays for many commercially important species. Particularly with the SNP discovery through whole genome resequencing approaches, and improvement of genotyping techniques, commercially accessible assays have grown from thousands of markers, to hundreds of thousands markers for some species [e.g. salmon $130 \mathrm{~K}$ [36], catfish $250 \mathrm{~K}$ [37] and $690 \mathrm{~K}$ [38], common carp $250 \mathrm{~K}$ [39]]. While this is useful for some applications (e.g. genome wide association studies), in many situations, genotyping at this marker density is not economically feasible, or required to complete the desired analysis [40, 41]. For a number of species with a closed nucleus breeding design, lower density SNPs assays with a few thousand 
markers are now being developed for routine breeding applications for many species [e.g. cattle [42], chickens [43] and salmon [40]].

In this study, for P. monodon we identified that 4000 markers were sufficient for the design of the DArTcap array to ensure high concordance was obtained for GRM calculations when compared to those derived from all available filtered DArTseq markers (7542 SNPs; Fig. 2). For use of this assay in future projects we desired a correlation of $98 \%$, however, depending on the end use application and required genotyping costs, the marker density could be reduced to between 2000 and 2500 markers while still achieving approximately 95\% correlation to the 7542 DArTseq SNPs. Reducing marker densities further would result in the rapid decay of GRM correlations and is not advised for using in advanced genomic based breeding.

Similar studies have previously been completed for $L$. vannamei, and indicated 3000 SNPs were appropriate for GRM calculations [44]. Likewise, Wang [45] found 3200 SNPs for L. vannamei would be sufficient for accurate GRM calculations when assessing an independent commercial population. While the genome sizes of $P$. monodon and L. vannamei are similar, and karyotypes are identical [2.2 Gb vs $1.7 \mathrm{~Gb}$; 44 chromosomes; Guppy [46]], the lower required number of markers for GRMs in L. vannamei may be due to both studies utilising samples from established breeding programs (many generations removed from wild) with large full-sib/half-sib family structures rather than wild individuals from two distinct regions in this study [47]. Effectively, a large full-sib/half-sib family dataset structure requires fewer markers to completely tag each segregating portion of the genome, and as such results in lower variation in GRM estimates at lower marker densities [48-50]. In future studies it would be valuable to reassess the required density of markers for $P$. monodon stock after multiple generations of domestication. Species with smaller and/ or less complex (less polymorphic/repetitive elements) genomes or low effective population sizes may be able to use smaller arrays and should undertake a similar approach when designing an assay.

\section{SNP linkage disequilibrium}

While a small number of marker pairs were observed to have linkage disequilibrium (LD) greater than 0.8 , only one of these pairs was regarded as highly linked in both Northern Territory and East Australian Coast regions. Given the genetic distinction between regions (Fig. 5), it is expected that the patterns of LD across individuals from each region will be different as well, and markers in low LD in one population may be unlinked (or show a varied level of linkage) in another [51, 52]. As such, these markers were not removed from the assay.
Furthermore, unlike livestock species which have low effective populations sizes (with highly similar organisation of genomes between individuals), the majority of commercial $P$. monodon cultured in Australia are not yet distinctly separated from wild stock (Supp. Figure 2), and will exhibit a large degree of varied genome arrangement across various individuals. Further investigation is warranted, however, as patterns of LD, both across regions and within regions, will impact the ability to translate genomic selection models (both in GRMs and estimating SNP effects) across to commercial stocks derived from different regions $[41,53,54]$.

While not available at the time of SNP selection, an early draft assembly of the $P$. monodon genome has since been made available [55]. Mapping markers to this assembly could be an additional approach used to filter out loci by genome position; however, the assembly contains over four million contigs and as such, currently has insufficient contiguity to be informative for this approach. Further work is underway to improve the $P$. monodon genome assembly, and the distribution of SNPs in this assay should be assessed further when this is available.

\section{Assay performance}

The conversion rate of the selected probes into a successful assay was high, with $99 \%$ of the targeted 4236 markers returned. Furthermore the genotyping call rate across the individuals resubmitted for DArTcap genotyping was high $(92.40 \% \pm 2.75 \% \mathrm{SD})$, and has remained high in subsequent genotyping of independent samples from additional Australian populations (Western Australia) and commercial stock $(93.50 \% \pm 3.90 \%$ SD). Furthermore, while the average call rate obtained across all individuals $(93.05 \% \pm 3.51 \% \mathrm{SD})$ in this study with DArTcap genotyping is marginally lower than those achieved with solid state genotyping [e.g. 98.92\%, Illumina Infinium ShrimpLD-24; Jones [56]], it is markedly higher than call rates achieved through traditional de novo GBS approaches (e.g. $86 \%$ in raw DArTseq data for this study). The improvement in data quality obtained with DArTcap genotyping over de novo GBS is further evident after the removal of low confidence genotype calls from the DArTseq dataset as the call rate decreased to $66 \%$. Similarly, while the average MAF of the DArTcap SNPs $(0.23 \pm 0.15)$ was lower than reported by Jones [56] (0.37), the DArTcap assay holds sufficient 'informativeness' for routine applications including assigning parentage (Table 2) and assessing genomic relatedness (GRM; Fig. 4) or population structure (Fig. 5).

A secondary SNP (additional non-targeted polymorphism with the target sequence) was obtained for a number of the probes and for routine application should be removed (as these are in complete linkage with target 
SNP); however they may represent additional valuable information if combined into RAD-tag haplotypes [57], or when the assay is applied to populations where the allele frequency of the target SNP is not as informative as the original tested populations (e.g. rare or fixed). Given the global value of the $P$. monodon industry it would be of interest to further assess the utility of this assay across potentially divergent international populations and commercial stocks.

The sequencing effort applied to each sample was markedly reduced for each individual (from 2.5 million to 670,610 reads per individual total) in DArTcap over DArTseq; however the average read depth obtained for each genotype call was higher (80.03 reads in DArTcap vs 17.01 reads DArTSeq), substantially increasing the confidence and accuracy of genotype calls. Furthermore, given sequencing costs are the most substantial cost associated with GBS, being able to reduce the total allocation required to obtain robust data is critical for routine applications involving the genotyping of thousands of individuals annually as required by breeding programs or when undertaking genome-wide association studies. Through utilising DArTcap genotyping over DArTseq genotyping, three times the total number of individuals can be assessed for an equivalent fixed financial investment (accounting for fixed per sample costs such as DNA extraction, service provider labour).

\section{Assay utilisation \\ Parentage assignment}

The ability to undertake parentage assignment remains a fundamental requirement for the vast majority of commercial farms and research end users. In many aquaculture production systems, including the $P$. monodon industry, progeny are produced from mass spawning events of multiple broodstock, with progeny of many families being reared communally from fertilisation onwards. Quite often it is not possible to manually tag or separate family lines. As a result, genotyping progeny and broodstock, and assigning parentage through molecular means is essential as it enables the recovery of pedigree information and ensures breeding programs are managed effectively $[27,58]$.

The parentage assignment success rate obtained in this study when using the 4194 DArTcap was high (up to $98.61 \%$ ), and further validates the utility of this assay more broadly. False assignment was not observed within any parental assignment analysis over the three datasets and various genotyping error rates, and would be highly unusual given the statistical approach employed in Colony [30]. Furthermore, false exclusions rates were low across all analyses (with the exception of DArTcap parentage run at $1 \%$ error) with only a single individual performing consistently poorly irrespective of SNP set or other analysis variables. With the 4194 DArTcap SNP sets, the rate of false exclusions were sensitive to the estimated genotype error rate included in the analysis, however, this is well known [59] and can be easily accommodated for in analysis.

Unlike in both DArTseq datasets, individual Mendelian inheritance errors (single genotype calls) were not silenced in the DArTcap dataset, and will have contributed to a large degree of the variation in exclusion rates seen between assays at each estimated genotyping error rate [60]. This approach was taken for validation purposes, as we wanted to only utilise prior parentage knowledge to valid assignments, rather than to assist in the analysis itself (e.g. including a single parent known). This provided the best equivalent to circumstances seen in routine commercial situations where no parental data can be linked in advance.

Depending on marker polymorphism and the relatedness of individuals, small arrays of 80-200 SNPs are often sufficient for parentage assignment [60, 61]. In previous studies using solid state technology (Sequenom) rather than GBS, Sellars [26] found similar assignment success (>95\%) to those achieved in this study are possible by using assays including 122 SNPs on eighth generation domesticated $P$. monodon stock. The parentage assignment success of the 122 SNPs assay provided by Sellars [26] was higher when compared to previous approaches with 12 or 13 microsatellites [62,63]. Further investigation of specific parentage subsets within the DArTcap assay should be explored to allow substantial reduction in time and computational requirement involved in assignment. To date, no direct studies of parentage assignment using GBS for pooled samples are available, but should be explored to further reduce genotyping expenses [46, 64].

\section{Genomic relationship calculations}

While comparisons of genomic relationships between the two assay types have been discussed, for the purposes of validating the assay we further tested the ability of the GRMs calculated from the DArTcap array across an independent communally reared commercial progeny cohort. When visualising the genomic relationship matrices with a dendrogram clustered heat map (Fig. 4), it is evident that full-sib and half-sib relationships can be separated into their respective family groupings, even in the absence of reference to any parental genotype or manual pedigree information. Furthermore a range of cryptic relationships are evident in the group of individuals tested, whereby individuals share either higher or lower relatedness values than expected in the traditional discrete pedigree relationships (i.e. $0=$ unrelated, $0.25=$ half-sib, $0.5=$ full sib). 
For genomic selection applications, it is not necessary in all circumstances to be able to obtain full parentage information (i.e. parent - progeny relationship), but instead determine an accurate estimate of the relatedness of individuals included in both the testing and training populations datasets [49]. Under circumstances where the true relationship between two individuals is inaccurate, their relative value or merit in breeding programs (EBV or GEBV) can be also incorrectly estimated [49]. The increase in selection accuracy by utilising GRMs has been well studied in terrestrial agriculture species [65] and has seen increasing attention in aquaculture breeding programs $[40,66-68]$. When compared to mass selection approaches that are currently available for the black tiger shrimp aquaculture industry, utilising GRMs simultaneously allows increased accuracy of selection [69], and further mitigation of inbreeding [e.g. optimised contribution selection [70];].

\section{Population segregation with DArTcap}

The ability to identify cryptic genetic sub-structuring within populations or groups can be useful to not only identify unique genetic stocks for founder selection [71], but also to trace captive lineages back to wild source populations [72], or identify escapees from aquaculture facilities [73].

Currently translocation of $P$. monodon broodstock occurs under permits between the Northern Territory and the East Australia Coast. Northern Territory broodstock in particular are captured from wild fisheries, and spawned in contained facilities in East Australia Coast region. The DArTcap assay was successfully able to reassign Northern Territory, East Australia Coast and Western Australia samples to their true population of origin (Fig. 5). If an escape event was suspected, this assay could be applied successfully to trace sampled individuals (broodstock or progeny) back to their source population (Sup Figure 2) This approach has been explored extensively in salmonoid aquaculture [74, 75], as well as in other species (e.g. turbot [76]).

\section{Considerations for future filtering of GBS and SNP selection GBS assays}

While genotype-by-sequencing approaches are promising and are being applied across multiple species $[1,9$, 46], there are a number of considerations that need to be addressed. Compared to solid state genotyping on fixed arrays (i.e. Illumina, Affymetrix), GBS approaches usually yield lower genotyping accuracies due to the nature of low read coverage in some SNPs. Filtering tailored to specific datasets can remove genotype calls with lower accuracies which in turn increase the accuracy and quality of the dataset as a whole, however, overly aggressive filtering can also remove large extents of data, much of which is of sufficient quality for downstream analysis [9]. Insufficient filtering thresholds, or absence of specific criteria themselves (e.G. minimum read depth) can result in spurious genotypes persisting in the dataset and being used in subsequent downstream analyses [77-79].

During the design of the $4 \mathrm{k}$ DARTcap array, we aimed to use balanced SNP filtering thresholds that retained as much data as possible, while removing the SNPs and genotype calls for which we had low confidence. A number of major genotyping performance metrics were explored including call rate, minor allele frequency, Mendelian error rates and Hardy-Weinberg deviations. It is interesting to note that a number of these metrics are intrinsically linked [e.g. call rate and read depth, read depth and MI error, repeatability and read depth [9];]. In addition, when transitioning from DArTseq to DArTcap procedures, a number of metrics (Call rate, read depth, reduced MI errors) were expected to improve dramatically $[13,16]$.

As such we placed the highest emphasis on marker minor allele frequency and call rate to ensure the allele frequencies remain high enough for use across multiple generations of selective breeding, and the markers were present in as many genotyped individuals as possible (avoiding population specific markers and those that were difficult to sequence). With reduced error in mind, markers showing on average higher read depths and higher repeatability of genotype calls (indicating consistent sequencing both between and within individuals) were preferentially ranked well. Furthermore, SNPs with low levels of MI error and small deviations from HWE were expected to improve with increased sequencing depth of DArTcap genotyping, and comparatively were not ranked down aggressively, instead the SNPs representing the majority of MI errors (>9\%) and significant deviations from HWE in both populations were removed earlier.

Markers with excessive read depth (e.g. > 200 reads/ genotype) should also be avoided, as this may indicate two markers erroneously being called together from paralogs (over-clustered), or located in highly repetitive regions [41, 77]. Filtering these markers was overlooked during the assay design phase of this study. While we removed secondary loci from each RAD-tag, it is also advisable to avoid highly polymorphic RAD-tags as the clustering undertaken during the bioinformatics processes involved in SNP calling becomes inherently more complex, and can lead to over-splitting of markers due incorrect phasing of alleles [41, 79]. Lastly, when selecting RAD-tags consideration should be taken to ensure the target SNP is not located near the fragment end to avoid issues with tag binding. Similarly, selection criteria for inclusion markers within other genotyping 
technologies should be considered where possible. For instance, SNP position is critical in Illumina probe design, and may hinder the transferability of the markers developed in this study to other genotyping assays.

\section{Conclusions}

A method to routinely genotype thousands of individuals is required to undertake advanced selective breeding in black tiger shrimp. This study described the development and validation of a crucial genotyping resource, which by utilising target capture genotype by sequencing (DArTcap) can for the first time be cost-effectively utilised in routinely commercial breeding $(<\$ 15$ AUD per sample). This assay containing 4194 SNPs, simultaneously provides the ability reconstruct the pedigree of communally reared families, while also being able to accurately calculate the cryptic genomic relationships between individuals. Furthermore, this assay will facilitate genome-wide association studies, linkage mapping, and unlocks the ability to undertake genomic selection black tiger shrimp.

\section{Methods}

\section{Sample collection and DNA isolation}

To develop informative genotyping assays, it is necessary to compile as diverse discovery population as practically possible while encapsulating the range of individuals to be utilized within the industry breeding programs. As such, samples were collected from a number of sources across the natural range of Australian P. monodon ( $n=$ 264; Supplementary Figure 1) and from 1st and 2nd generation farm stock ( $n=165$ and $n=231$ respectively). Given the aims and design of this study, a priori sample size calculations were not appropriate. Farm stock were provided for sampling by Seafarms Group Ltd. as part of routine operational practises, while individuals from natural populations were obtained from CSIRO fishery surveys and were collected within the guidelines of appropriate permits for each location. All individuals were euthanized upon collection through immersion in an ice-slurry. Pleopod tissue or whole post larvae were collected and stored in ethanol or RNA-later at $-20^{\circ} \mathrm{C}$ until extraction. Genomic DNA was extracted either following the CTAB extraction protocol or MagJET Genomic DNA Kit ([80] and ThermoFisher Scientific). Genomic DNA was purified using Sephadex G-50 (GE Healthcare Life Sciences 2000) and visualised on 0.8\% agarose gel to ensure quality and quantity were sufficient.

\section{Genome-wide SNP discovery}

In the absence of existing published GBS datasets for $P$. monodon, a reduced-representation genome by sequencing (GBS) approach, DArTseq, was employed for SNP discovery through a service provider, Diversity Arrays Technologies (DArT [4];). High quality DNA was provided to DArT to identify SNP markers through a restriction digest reduced-representation based sequencing strategy as described in [4, 81]. Briefly, DNA from 660 shrimp underwent a restriction digest using Pstl and Hpall restriction enzymes and unique proprietary barcodes for each sample were ligated to size-selected DNA. Samples that demonstrated non-uniform digestion patterns were excluded from library preparation $(n=10)$. To allow a measure of technical repeatability in genotype calls and library preparation, 147 random replicates (23\%) were included within the library preparation process. Equimolar amounts of barcoded samples were pooled, with 94 samples forming a single pool, before sequencing cluster preparation on the Illumina c-Bot bridge PCR system. Libraries were sequenced on three flow cell lanes on an Illumina HiSeq2500 to provide an average of 2.5 million raw reads per individual.

Reads with low sequence quality scores $(\mathrm{Q}<25)$ were eliminated and SNP calling was completed using the KDcompute pipeline (DArT [81];). Following this, both monomorphic loci and sequences associated with species other than P. monodon (human, bacterial etc.) were excluded from the data set.

\section{Marker quality control}

To ensure only high quality markers were included in the final target capture genotyping assay, multiple steps of filtering were applied to the dataset before the remaining SNPs were ranked on quality and level of polymorphism prior to final selection. Custom python scripts were developed to efficiently handle the datasets provided by DArT (github.com/esteinig/dartQC) and undertake preliminary SNP filtering for quality. Briefly, genotype calls were silenced on the basis of low cumulative raw read depth (i.e. with sum of reads for both alleles less than five), before SNPs with minor allele frequency (MAF) less than 0.02, call rate less than 50\% or less than $90 \%$ repeatability were removed. Next, SNP redundancy filtering was undertaken, whereby sequence clusters (RAD-tag or clones) were identified using the CD-HIT clustering algorithm at 95\% identity [82], and then the SNP with the highest MAF within each sequence cluster was retained. This redundancy filtering was undertaken to ensure that overrepresentation of specific areas of the genome did not occur, as this may introduce bias into future genomic analyses.

Hardy Weinberg Equilibrium (HWE) deviations were calculated within PLINK [83] utilizing discrete datasets for the East Coast $(n=132)$ and Northern Territory stocks $(n=125)$ (Table 1$)$. SNPs identified to significantly deviate $(p<0.0001)$ from HWE in both populations were removed. Known parental trio relationships 
(81, progeny-dam-sire) were utilised to identify SNPs that displayed Mendelian Inheritance (MI) errors using PLINK [83]. MI errors were investigated on an individual SNP call basis as these could be due to incorrect genotype calls from sequencing error, or insufficient read depth [84]. SNPs with high levels of aberrant MI errors $(>9 \%)$ not associated with sequence coverage errors were removed.

\section{SNP selection and assay design}

To determine the number of markers required to accurately obtain GRMs in P. monodon, calculations were undertaken using a similar approach to Rolf [42]. GRMs calculated from increasing densities of markers were compared to a GRM estimated from all 7542 SNPs (G). The programs $\mathrm{R} v 3.4 .1$ and PLINK v1.9 [83] were used to create randomly selected marker subsets $(n=100$, $200,500,1000,1500,2500,3000,3500,4000,4500$, $5000,5500,6000,6500,7000)$, with replacement, from the pool of 7542 SNPs available. For each marker subset (n), 50 random replicates $(i)$ were generated and a GRM $\left(\mathbf{G}_{\boldsymbol{n}}\right)$ was estimated using GCTA $v 1.91 .7 b$ [85]. Correlations were then drawn between the pairwise relatedness of 650 individuals (Table 1) produced in each $\mathbf{G}_{\boldsymbol{n} \boldsymbol{i}}$ GRM and the corresponding relatedness values of $\mathbf{G}$. Average pairwise relatedness values across the 50 replicates for each marker subset were calculated before correlation plots were produced using the R package ggplot2 [86]. Such GRM analysis (detailed below) indicated that a panel of 4000 SNPs was able to produce a GRM with an $r^{2}$ correlation of 0.98 to the full 7542 array. Therefore, this defined the target size of the DArTcap sub-array to be developed.

To prioritise the selection of the highest quality SNPs in the final assay, a custom quality score (QC score) was developed to rank each SNP by a range of SNP quality metrics (Eq. 1). Metrics included were, call rate $(C R)$ which ranged from 0 to 1 with a weighting of 1.4; minor allele frequency $(M A F)$ ranging from 0 to 0.5 with a weighting of 2.4; average repeatability (Rep_Avg) ranging from 0 to 1 ; standardised read depth $(R D)$ whereby the average read depth of the SNP was divided by the largest read depth observed to give a proportion between 0 and 1; Hardy Weinberg Equilibrium deviation $(H W E)$ as a percentage; and Mendelian inheritance error rate $(M I)$.

$$
\begin{aligned}
Q C \text { score }= & (C R \times 1.4)+(M A F \times 2.4) \\
& +R e p \_A v g+R D-\frac{(H W E)}{10}-M I
\end{aligned}
$$

A total of 4236 highest ranked QC score SNPs were selected for the $4 \mathrm{~K}$ genotyping assay allowing for some redundancy for marker drop out (cross probe affinity, probe failure) during probe synthesis. Furthermore, a
GRM was calculated using the 4236 subset of markers and compared to the full 7542 markers to ensure concordance was maintained. Selected sequences were provided to DArT, and DArTcap probes synthesized (MYbaits ${ }^{\circ}$, MYcroarray $^{\circ}$ ) for testing.

\section{Linkage disequilibrium}

To approximate the distribution and independence of markers across the genome, linkage disequilibrium (LD) was calculated across the complete datasets of 7542 SNPs and 4236 DArTseq SNPs with all available samples in PLINK [83]. Similarly, population specific LD was calculated with samples from the Northern Territory $(n=$ $125)$ and East Australian Coast $(n=132)$ regions independently. Pairwise LD values between loci greater than $>0.2$ were reported, and then compared between Northern Territory and East Australian Coast regions to identify if any markers were consistently in complete $\left(r^{2}=1\right)$ or high LD $\left(r^{2}>0.8\right)$.

\section{Assay validation}

Validation of the DArTcap probes involved the regenotyping of a subset of 251 individuals from the discovery population (Table 1). DArTcap follows similar methodology to DArTseq, however, it involves an additional magnetic bead hybridization step (Dynabeads, MyOne) that utilises the DArTcap probes to capture and enrich the 4236 target SNP sequences before being put forward for sequencing. Sequencing of the targeted (DArTcap) library and preliminary sequence data quality control was identical to the DArTseq procedure described above. A minimum of $8 \%$ technical replicates (i.e. 8 samples per 94 well plate) were included to provide a measure of SNP repeatability.

In addition to sequencing a representation of the DArTseq discovery population, two groups of additional novel individuals (19 individuals from Nickol Bay, Western Australia, and 282 additional commercial progeny; Table 1) were also included in the DArTcap sequencing effort as independent datasets for validation and analysis. In order to evaluate the quality and integrity of the DArTcap assay, comparisons were drawn between SNP metrics (i.e. call rate, read depth, Mendelian inheritance errors) produced by the two GBS methods (DArTseq and DArTcap). In addition, parentage analysis, GRM calculations and sample relatedness were also assessed with the 7542 DArTseq SNP dataset, the 4236 DArTseq SNP dataset and the final 4194. DArTcap SNP dataset to validate the utility of the finalized assay.

\section{Parentage assignment}

As parentage assignment is a fundamental requirement for many genotyping assays, power to assign parentage was tested across three SNP datasets (the 75,442 filtered 
DArTseq SNPs, the 4236 DArTseq SNPs selected for assay design and the 4194. DArTcap SNPs). A total of 46 progeny and 56 broodstock with known parent-progeny relationships were available for genotyping on both the DArTseq and DArTcap platforms. A number of additional broodstock with known parent-progeny relationships had insufficient DNA when undertaking DArTcap genotyping and were excluded. For these 72 family pairwise relationships, parentage assignment was undertaken in the program Colony [30]. Repeated analyses with estimated genotyping error rates ranging from strict (1\%), to moderate $(5 \%)$, to conservative $(10 \%)$ were completed to account for undefined genotyping error rates across the three SNP datasets [87]. Since the progeny were the first generation of wild broodstock pairings, inbreeding was not included. Prior sib-ship assumptions were excluded allowing for highly skewed family sizes commonly observed in mass-spawning aquaculture systems [58]. Likewise maternal and paternal polygamy were allowed to account for potential of half-sib breeding designs that utilise artificial insemination. All analyses were completed with the 'long run', 'high precision' and 'fulllikelihood' options within Colony. The two types of parentage assignment errors; a) incorrect exclusion of a true parent, and b) assignment of an untrue parent, were determined for each assignment analysis.

\section{Calculating relatedness and identifying genetic structure}

To confirm the estimates of relatedness obtained from DArTcap genotyping (4129 SNPs) were concordant with those of the selected 4236 DArTseq markers, a GRM was calculated in GCTA v1.91.7b for both datasets and all common individuals $(n=195)$ in the two datasets were compared..

To provide a complementary approach to visually assess the utility of GRMs derived from DArTcap genotyping, GRMs and dendrograms were calculated for an independent set of G2 samples $(n=272$; Table 1$)$ and then plotted as a heat-map in $\mathrm{R}$ with the package 'heatmaply' [88].

To confirm the DArTcap assay retained sufficient informativeness to distinguish between individuals from distinct populations, we completed discriminant analysis of principal components (DAPC) using the $\mathrm{R}$ package adegenet [89]. We completed this analysis with three subsets of the 4194 DArTcap SNP dataset. Firstly, all available individuals $(n=418)$ secondly retaining only wild sourced broodstock and wild samples and excluding all G2 individuals $(n=168)$ to ensure closely related individuals were not influencing the analysis, and lastly with only an independent set of G2 samples $(n=272)$ to assess the ability to discriminate between family lines. Concordance of sample placement within source populations was assessed.

\section{Supplementary information}

Supplementary information accompanies this paper at https://doi.org/10. 1186/s12864-020-06960-w.

\section{Additional file 1: Supplementary Figure 1. Penaeus monodon} distribution across Australia (light grey), approximate locations of current pond based farming operations (dark grey), and location of samples included in DARTcap 'discovery' populations (1 - Joseph Bonaparte Bay $(n=34), 2$ - Tiwi Islands $(n=56), 3$ - Gulf of Carpentaria $(n=43), 4$ Bramston Beach $(n=60), 5$ - Etty Bay $(n=50), 6$ - Townsville $(n=22)$ and 7 - Commercial Farm site $(n=394)$. Supplementary Figure 2.

Clustering of samples based upon genetic similarity shown through discriminant analysis of principle components (DAPC). PC1 and PC2 are shown on the $x$ and $y$ axis respectively. (A) Including all samples ( $n=$ 418). (B) Including only second generation (G2) individuals $(n=272)$ obtained from routine commercial spawning.

\section{Abbreviations}

AFLPs: Amplified fragment length polymorphisms; CTAB: Cetyl trimethylammonium bromide; DAPC: Discriminant analysis of principle components; DArT: Diversity Arrays Technologies; DNA: Deoxyribonucleic acid; EBV: Estimated breeding value; G1/G2: Generation 1 and 2;

Gb: Gigabase; GBS: Genotype-by-sequencing; GEBV: Genomic estimated breeding value; GRM: Genomic relationship matrix; GS: Genomic selection; GWAS: Genome wide association studies; HWE: Hardy-Weinberg equilibrium; LD: Linkage disequilibrium; MAF: Minor allele frequency; MI: Mendialian inheritance; QC: Quality control; QTL: Quantitative trait locus; RADSeq: Restriction site Associated DNA Sequencing; SD: Standard deviation; SNP: Single nucleotide polymorphism; PC: Principle components

\section{Acknowledgements}

The authors are grateful for assistance provided by employees of Diversity Array Technologies in the development of the custom genotyping assay, and Cheryl Tan for preparing samples for submission. The authors would also like to thank Seafarms Group Ltd. in Queensland for providing access to animals, and supporting harvesting and collection of commercial samples.

\section{Authors' contributions}

KRZ, DRJ, HWR and JLG conceived the study. JLG analysed the data and prepared the manuscript and figures. DBJ, SRK, MSK, NMW, HWR, KRZ, AL and EJS provided guidance undertaking of analysis and assisted with interpretation of results. MJS facilitated the collection of wild samples. NMW managed the collection of commercial animals and associated breeding information. EJS wrote custom python scripts used throughout analysis. DBJ, SRK, MJS, DRJ, KRZ assisted with early drafting of the manuscript. All authors read and approved the manuscript.

\section{Funding}

This project was supported by The Australian Research Council Industrial Transformation Research Hub for Advanced Prawn Breeding (IH130200013). The first author was supported by a scholarship from the Australian Government Department of Education and Training. The funding bodies had no role in the design of the study or collection, analysis and interpretation of the data.

\section{Availability of data and materials}

The DArTseq and DArTcap datasets files generated during the current study, including read counts and genotypes, are available on the DRYAD data repository (https://doi.org/10.5061/dryad.qz612jmc8).

\section{Ethics approval and consent to participate}

Under the Australian Code for the Care and use of animals for scientific purposes, institutional ethics approval involving experimentation for nonvertebrates (excluding cephalopods) is not required - thus for the current study animal ethics was not required. (https://www.nhmrc.gov.au/about-us/ publications/australian-code-care-and-use-animals-scientific-purposes\#blockviews-block-file-attachments-content-block-1). 


\section{Competing interests}

The authors declare that they have no competing interests.

\begin{abstract}
Author details
'Australian Research Council Industrial Transformation Research Hub for Advanced Prawn Breeding, James Cook University, Townsville, QLD 4811, Australia. ${ }^{2}$ Centre for Sustainable Tropical Fisheries and Aquaculture, College of Science and Engineering, James Cook University, Townsville, QLD 4811, Australia. ${ }^{3}$ Sydney School of Veterinary Science, Faculty of Science, The University of Sydney, Camden, NSW 2570, Australia. ${ }^{4}$ CSIRO Agriculture \& Food, Integrated Sustainable Aquaculture Production Program, Queensland Bioscience Precinct, St Lucia, QLD 4067, Australia. ${ }^{5}$ Present Address: Genics Pty Ltd, Research Road, St Lucia, QLD 4011, Australia. ${ }^{6}$ Australian Institute of Tropical Health and Medicine, James Cook University, Townsville, QLD 4811, Australia. ${ }^{7}$ Tropical Futures Institute, James Cook University, Singapore, Singapore.
\end{abstract}

Received: 9 January 2020 Accepted: 29 July 2020

Published online: 05 August 2020

\section{References}

1. Robledo D, Palaiokostas C, Bargelloni L, Martínez P, Houston R. Applications of genotyping by sequencing in aquaculture breeding and genetics. Rev Aquac. 2017;10:1-13. https://doi.org/10.1111/raq.12193.

2. Miller MR, Dunham JP, Amores A, Cresko WA, Johnson EA. Rapid and costeffective polymorphism identification and genotyping using restriction site associated DNA (RAD) markers. Genome Res. 2007;17(2):240-8.

3. Baird NA, Etter PD, Atwood TS, Currey MC, Shiver AL, Lewis ZA, et al. Rapid SNP discovery and genetic mapping using sequenced RAD markers. PLoS One. 2008;3(10):e3376.

4. Sansaloni C, Petroli C, Jaccoud D, Carling J, Detering F, Grattapaglia D, et al. Diversity arrays technology (DArT) and next-generation sequencing combined: genome-wide, high throughput, highly informative genotyping for molecular breeding of Eucalyptus. BMC Proc. 2011;5(7):54.

5. Peterson BK, Weber JN, Kay EH, Fisher HS, Hoekstra HE. Double digest RADseq: an inexpensive method for De novo SNP discovery and genotyping in model and non-model species. PLoS One. 2012;7(5):e37135.

6. Wang S, Meyer E, McKay JK, Matz MV. 2b-RAD: a simple and flexible method for genome-wide genotyping. Nat Methods. 2012;9(8):808-10.

7. Toonen RJ, Puritz JB, Forsman ZH, Whitney $J \mathrm{~L}$, Fernandez-Silva I, Andrews KR, et al. ezRAD: a simplified method for genomic genotyping in nonmodel organisms. PeerJ. 2013;1:e203.

8. Graham CF, Glenn TC, MCArthur AG, Boreham DR, Kieran T, Lance S, et al. Impacts of degraded DNA on restriction enzyme associated DNA sequencing (RADSeq). Mol Ecol Resour. 2015;15(6):1304-15.

9. Andrews KR, Good JM, Miller MR, Luikart G, Hohenlohe PA. Harnessing the power of RADseq for ecological and evolutionary genomics. Nat Rev Genet. 2016;17(2):81-92.

10. Li Y-H, Wang H-P. Advances of genotyping-by-sequencing in fisheries and aquaculture. Rev Fish Biol Fish. 2017.

11. Puritz JB, Matz MV, Toonen RJ, Weber JN, Bolnick DI, Bird CE. Demystifying the RAD fad. Mol Ecol. 2014;23(24):5937-42.

12. Jones MR, Good JM. Targeted capture in evolutionary and ecological genomics. Mol Ecol. 2016;25(1):185-202.

13. Ali OA, O'Rourke SM, Amish SJ, Meek MH, Luikart G, Jeffres C, et al. RAD capture (rapture): flexible and efficient sequence-based genotyping. Genetics. 2016;202(2):389-400.

14. Suchan T, Pitteloud C, Gerasimova NS, Kostikova A, Schmid S, Arrigo N, et al. Hybridization capture using RAD probes (hyRAD), a new tool for performing genomic analyses on collection specimens. PLoS One. 2016;11(3):e0151651.

15. Holtz Y, Ardisson M, Ranwez V, Besnard A, Leroy P, Poux G, et al. Genotyping by sequencing using specific allelic capture to build a highdensity genetic map of durum Wheat. PLoS One. 2016;11(5):e0154609.

16. Hoffberg SL, Kieran TJ, Catchen JM, Devault A, Faircloth BC, Mauricio R, et al. RADcap: sequence capture of dual-digest RADseq libraries with identifiable duplicates and reduced missing data. Mol Ecol Resour. 2016;16(5):1264-78.

17. FishStat Plus - Universal software for fishery statistical time series. [Internet]. 2017 [cited 10 June 2017].

18. Nielsen HM, Sonesson AK, Meuwissen THE. Optimum contribution selection using traditional best linear unbiased prediction and genomic breeding values in aquaculture breeding schemes. J Anim Sci. 2011;89:630-8.
19. Norman-López A, Sellars MJ, Pascoe S, Coman GJ, Murphy B, Moore N, et al. Productivity benefits of selectively breeding black Tiger shrimp (Penaeus monodon) in Australia. Aquac Res. 2016;47(10):3287-96.

20. Wang Q, Yu Y, Yuan J, Zhang X, Huang H, Li F, et al. Effects of marker density and population structure on the genomic prediction accuracy for growth trait in Pacific white shrimp Litopenaeus vannamei. BMC Genet. 2017;18(1):45.

21. Zenger KR, Khatkar MS, Jerry DR, Raadsma HW. The next wave in selective breeding: implementing genomic selection in aquaculture. Townsville: AAABG; 2017.

22. Brooker AL, Benzie JAH, Blair D, Versini J-J. Population structure of the giant tiger prawn Penaeus monodon in Australian waters, determined using microsatellite markers. Mar Biol. 2000;136(1):149-57.

23. You EM, Chiu TS, Liu KF, Tassanakajon A, Klinbunga S, Triwitayakorn K, et al. Microsatellite and mitochondrial haplotype diversity reveals population differentiation in the tiger shrimp (Penaeus monodon) in the indo-Pacific region. Anim Genet. 2008;39(3):267-77.

24. Supungul $P$, Sootanan $P$, Klinbunga S, Kamonrat W, Jarayabhand $P$, Tassanakajon A. Microsatellite polymorphism and the population structure of the black Tiger shrimp (Penaeus monodon) in Thailand. Mar Biotechnol. 2000;2(4):339-47.

25. Wilson K, Li YT, Whan V, Lehnert S, Byrne K, Moore S, et al. Genetic mapping of the black tiger shrimp Penaeus monodon with amplified fragment length polymorphism. Aquaculture. 2002;204(3-4):297-309.

26. Sellars MJ, Dierens L, McWilliam S, Little B, Murphy B, Coman GJ, et al. Comparison of microsatellite and SNP DNA markers for pedigree assignment in black Tiger shrimp, Penaeus monodon. Aquaculture Res. 2014;45(3):417-26.

27. Jerry DR, Preston NP, Crocos PJ, Keys S, Meadows JR, Li Y. Parentage determination of Kuruma shrimp Penaeus (Marsupenaeus) japonicus using microsatellite markers (bate). Aquaculture. 2004;235(1):237-47.

28. Baranski M, Gopikrishna G, Robinson NA, Katneni VK, Shekhar MS, Shanmugakarthik J, et al. The development of a high density linkage map for black tiger shrimp (Penaeus monodon) based on CSNPs. PLoS One. 2014;9(1):e85413.

29. Wang J. Marker-based estimates of relatedness and inbreeding coefficients: an assessment of current methods. J Evol Biol. 2014;27(3):518-30.

30. Jones OR, Wang J. COLONY: a program for parentage and sibship inference from multilocus genotype data. Mol Ecol Resour. 2010;10(3):551-5.

31. Makgahlela ML, Strandén I, Nielsen US, Sillanpää MJ, Mäntysaari EA. The estimation of genomic relationships using breedwise allele frequencies among animals in multibreed populations. J Dairy Sci. 2013;96(8):5364-75.

32. Dodds KG, McEwan JC, Brauning R, Anderson RM, van Stijn TC, Kristjánsson $\mathrm{T}$, et al. Construction of relatedness matrices using genotyping-bysequencing data. BMC Genomics. 2015;16(1):1047.

33. Yu Y, Zhang X, Yuan J, Li F, Chen X, Zhao Y, et al. Genome survey and highdensity genetic map construction provide genomic and genetic resources for the Pacific white shrimp Litopenaeus vannamei. Sci Rep. 2015;5:15612.

34. Guo L, Xu Y-H, Zhang N, Zhou F-L, Huang J-H, Liu B-S, et al. A High-Density Genetic Linkage Map and QTL Mapping for Sex in Black Tiger Shrimp (Penaeus monodon). Frontiers in Genetics. 2019;10(326):1-9.

35. Robinson NA, Gopikrishna G, Baranski M, Katneni VK, Shekhar MS, Shanmugakarthik J, et al. QTL for white spot syndrome virus resistance and the sex-determining locus in the Indian black tiger shrimp (Penaeus monodon). BMC Genomics. 2014;15(1):731

36. Houston RD, Taggart JB, Cézard T, Bekaert M, Lowe NR, Downing A, et al. Development and validation of a high density SNP genotyping array for Atlantic salmon (Salmo salar). BMC Genomics. 2014;15(1):90.

37. Liu S, Sun L, Li Y, Sun F, Jiang Y, Zhang Y, et al. Development of the catfish $250 \mathrm{~K}$ SNP array for genome-wide association studies. BMC Res Notes. 2014;7(1):135

38. Zeng Q, Fu Q, Li Y, Waldbieser G, Bosworth B, Liu S, et al. Development of a 690 K SNP array in catfish and its application for genetic mapping and validation of the reference genome sequence. Sci Rep. 2017;7:1-14.

39. Xu J, Zhao Z, Zhang X, Zheng X, Li J, Jiang Y, et al. Development and evaluation of the first high-throughput SNP array for common carp (Cyprinus carpio). BMC Genomics. 2014;15(1):307.

40. Tsai H-Y, Matika O, Edwards SM. Antolín-Sánchez R, Hamilton A, Guy DR, et al. Genotype Imputation To Improve the Cost-Efficiency of Genomic Selection in Farmed Atlantic Salmon. G3: Genes|Genomes|Genetics. 2017;7(4):1377-83.

41. Liu ZJ. Bioinformatics in aquaculture: principles and methods: John Wiley \& Sons; 2017.

42. Rolf MM, Taylor JF, Schnabel RD, McKay SD, McClure MC, Northcutt SL, et al. Impact of reduced marker set estimation of genomic relationship matrices on genomic selection for feed efficiency in Angus cattle. BMC Genet. 2010;11(1):24 
43. Reverter A, Li Y, George A, Henshall J, Sapp R, Okimoto R, et al. Deviations around kinship expectations at various SNP marker densities in a population of broiler chicken. Proc Assoc Advmt Anim Breed Genet. 2017;22:249-52.

44. Jones DB, Zenger KR, Khatkar MS, Raadsma HW, HAMvd S, Prochaska J, et al. In: Genetics AftAoABa, editor. development of a low-density commercial genotyping array for the white legged shrimp, Litopenaeus vannamei. Townsville: AAABG; 2017.

45. Wang Q, Yu Y, Li F, Zhang X, Xiang J. Predictive ability of genomic selection models for breeding value estimation on growth traits of Pacific white shrimp Litopenaeus vannamei. Chinese J Oceanol Limnol. 2016:35:1221-29.

46. Guppy JL, Jones DB, Jerry DR, Wade NM, Raadsma HW, Huerlimann R, et al. The state of 'omics' research for farmed penaeids: advances in research and impediments to industry utilisation. Frontiers in Genetics. 2018;9(282):1-27.

47. Slatkin M. Linkage disequilibrium--understanding the evolutionary past and mapping the medical future. Nat Rev Genet. 2008;9(6):477-85.

48. Solberg TR, Sonesson AK, Woolliams JA, Meuwissen THE. Genomic selection using different marker types and densities. J Anim Sci. 2008;86(10):2447-54.

49. Hayes BJ, Visscher PM, Goddard ME. Increased accuracy of artificial selection by using the realized relationship matrix. Genet Res. 2009;91(01):47-60.

50. Wang B, Sverdlov S, Thompson E. Efficient estimation of realized kinship from single nucleotide polymorphism genotypes. Genetics. 2017;205(3):1063-78.

51. Hinds DA, Stuve LL, Nilsen GB, Halperin E, Eskin E, Ballinger DG, et al. Wholegenome patterns of common DNA variation in three human populations. Science. 2005;307(5712):1072-9.

52. Porto-Neto LR, Kijas JW, Reverter A. The extent of linkage disequilibrium in beef cattle breeds using high-density SNP genotypes. Genet Sel Evol. 2014; 46(1):22.

53. Bolormaa S, Gore K, van der Werf JHJ, Hayes BJ, Daetwyler HD. Design of a low-density SNP chip for the main Australian sheep breeds and its effect on imputation and genomic prediction accuracy. Anim Genet. 2015;46(5):544-56.

54. Liu H, Zhou H, Wu Y, Li X, Zhao J, Zuo T, et al. The impact of genetic relationship and linkage disequilibrium on genomic selection. PLoS One. 2015;10(7):e0132379.

55. Yuan J, Zhang X, Liu C, Yu Y, Wei J, Li F, et al. Genomic resources and comparative analyses of two economical penaeid shrimp species, Marsupenaeus japonicus and Penaeus monodon. Mar Genomics. 2018;39:22-5.

56. Jones DB, Jerry DR, Khatkar MS, Raadsma HW, Hvd S, Prochaska J, et al. A comparative integrated gene-based linkage and locus ordering by linkage disequilibrium map for the Pacific white shrimp, Litopenaeus vannamei. Sci Rep. 2017;7(1):10360

57. Tinker NA, Bekele WA, Hattori J. Haplotag: Software for Haplotype-Based Genotyping-by-Sequencing Analysis. G3: Genes|Genomes|Genetics. 2016;6(4):857-63.

58. Foote A, Simma D, Khatkar M, Raadsma H, Guppy J, Coman G, et al. Considerations for Maintaining Family Diversity in Commercially Mass-Spawned Penaeid Shrimp: A Case Study on Penaeus monodon. Front Genet. 2019;10(1127):1-12.

59. Harrison HB, Saenz-Agudelo P, Planes S, Jones GP, Berumen ML. On minimizing assignment errors and the trade-off between false positives and negatives in parentage analysis. Mol Ecol. 2013;22(23):5738-42.

60. Vandeputte $M$, Haffray P. Parentage assignment with genomic markers: a major advance for understanding and exploiting genetic variation of quantitative traits in farmed aquatic animals. Front Genet. 2014;5(432):1-8.

61. Yue $\mathrm{GH}$, Xia $\mathrm{JH}$. Practical considerations of molecular parentage analysis in fish. J World Aquacult Soc. 2014;45(2):89-103.

62. Li Y, Wongprasert K, Shekhar M, Ryan J, Dierens L, Meadows J, et al. Development of two microsatellite multiplex systems for black tiger shrimp Penaeus monodon and its application in genetic diversity study for two populations. Aquaculture. 2007;266(1):279-88.

63. Dixon TJ, Coman GJ, Arnold SJ, Sellars MJ, Lyons RE, Dierens L, et al. Shifts in genetic diversity during domestication of black Tiger shrimp, Penaeus monodon, monitored using two multiplexed microsatellite systems. Aquaculture. 2008;283(1-4):1-6.

64. Henshall JM, Dierens L, Sellars MJ. Quantitative analysis of low-density SNP data for parentage assignment and estimation of family contributions to pooled samples. Genetics, Selection, Evolution : GSE. 2014;46(1):51.

65. Meuwissen T, Hayes B, Goddard M. Genomic selection: a paradigm shift in animal breeding. Animal frontiers. 2016;6(1):6-14.

66. Vallejo RL, Leeds TD, Gao G, Parsons JE, Martin KE, Evenhuis JP, et al. Genomic selection models double the accuracy of predicted breeding values for bacterial cold water disease resistance compared to a traditional pedigreebased model in rainbow trout aquaculture. Genet Sel Evol. 2017;49(1):17.
67. Robledo D, Matika O, Hamilton A, Houston RD. Genome-Wide Association and Genomic Selection for Resistance to Amoebic Gill Disease in Atlantic Salmon. G3: Genes|Genomes|Genetics. 2018;8(4):1195-203.

68. Yoshida GM, Carvalheiro R, Rodríguez FH, Lhorente JP, Yáñez JM. Single-step genomic evaluation improves accuracy of breeding value predictions for resistance to infectious pancreatic necrosis virus in rainbow trout. Genomics. 2019;111(2):127-32

69. Castillo-Juárez H, Campos-Montes GR, Caballero-Zamora A, Montaldo HH. Genetic improvement of Pacific white shrimp [Penaeus (Litopenaeus) vannamei]: perspectives for genomic selection. Front Genet. 2015;6(93):1-19.

70. Zenger KR, Khatkar MS, Jones DB, Khalilisamani N, Jerry DR, Raadsma HW. Genomic Selection in Aquaculture: Application, Limitations and Opportunities With Special Reference to Marine Shrimp and Pearl Oysters. Front Genet. 2019;9(693)

71. Sun L, Liu S, Wang R, Jiang Y, Zhang Y, Zhang J, et al. Identification and analysis of genome-wide SNPs provide insight into signatures of selection and domestication in channel catfish (Ictalurus punctatus). PLoS One. 2014;9(10):e109666.

72. Kijas JW, Gutierrez AP, Houston RD, McWilliam S, Bean TP, Soyano K, et al. Assessment of genetic diversity and population structure in cultured Australian Pacific oysters. Animal Genetics. 2019;0(0):686-94

73. Palti Y, Gao G, Liu S, Kent MP, Lien S, Miller MR, et al. The development and characterization of a 57K single nucleotide polymorphism array for rainbow trout. Mol Ecol Resour. 2015;15(3):662-72.

74. Gilbey J, Cauwelier E, Sampayo J, Matejusova I, Allan C, Graham J, et al. Identification of the farm of origin of Atlantic salmon smolt escapees in a freshwater Scottish loch using single-nucleotide polymorphic markers. ICES J Mar Sci. 2018;75(6):2182-92.

75. Pritchard VL, Erkinaro J, Kent MP, Niemelä E, Orell P, Lien S, et al. Single nucleotide polymorphisms to discriminate different classes of hybrid between wild Atlantic salmon and aquaculture escapees. Evol Appl. 2016:9(8):1017-31.

76. Prado FD, Vera M, Hermida M, Blanco A, Bouza C, Maes GE, et al. Tracing the genetic impact of farmed turbot Scophthalmus maximus on wild populations. Aquaculture Environment Interactions. 2018:10:447-63.

77. Davey JW, Cezard T, Fuentes-Utrilla P, Eland C, Gharbi K, Blaxter ML. Special features of RAD sequencing data: implications for genotyping. Mol Ecol. 2013;22(11):3151-64

78. Shafer ABA, Peart CR, Tusso S, Maayan I, Brelsford A, Wheat CW, et al. Bioinformatic processing of RAD-seq data dramatically impacts downstream population genetic inference. Methods Ecol Evol. 2017;8(8):907-17.

79. Díaz-Arce N, Rodríguez-Ezpeleta N, et al. Selecting RAD-Seq Data Analysis Parameters for Population Genetics: The More the Better?. Front Genet. 2019;10(533):1-10

80. Adamkewicz SL, Harasewych M. Systematics and biogeography of the genus Donax (Bivalvia: Donacidae) in eastern North America. Am Malacol Bull. 1996; 13:97-103.

81. Lind CE, Kilian A, Benzie JAH. Development of diversity arrays technology markers as a tool for rapid genomic assessment in Nile tilapia, Oreochromis niloticus. Animal Genet. 2017;48(3):362-4.

82. Fu L, Niu B, Zhu Z, Wu S, Li W. CD-HIT: accelerated for clustering the nextgeneration sequencing data. Bioinformatics. 2012;28(23):3150-2.

83. Purcell S, Neale B, Todd-Brown K, Thomas L, Ferreira MA, Bender D, et al. PLINK: a tool set for whole-genome association and population-based linkage analyses. Am J Hum Genet. 2007;81(3):559-75.

84. Chen N, Van Hout CV, Gottipati S, Clark AG. Using Mendelian inheritance to improve high-throughput SNP discovery. Genetics. 2014;198(3):847-57.

85. Yang J, Lee SH, Goddard ME, Visscher PM. GCTA: a tool for genome-wide complex trait analysis. Am J Hum Genet. 2011;88(1):76-82.

86. Wickham H. ggplot2: elegant graphics for data analysis. New York: SpringerVerlag; 2016.

87. Wang J. Pedigree reconstruction from poor quality genotype data. Heredity. 2019:122(6):719-28.

88. Galili T, O'Callaghan A, Sidi J, Sievert C. Heatmaply: an R package for creating interactive cluster heatmaps for online publishing. Bioinformatics. 2017;34(9):1600-2

89. Jombart T, Collins C. A tutorial for discriminant analysis of principal components (DAPC) using adegenet 2.0. 0. London: Imperial College London, MRC Centre for Outbreak Analysis and Modelling; 2015.

\section{Publisher's Note}

Springer Nature remains neutral with regard to jurisdictional claims in published maps and institutional affiliations. 